\section{Saúde infantil em áreas pobres: resultados de um estudo de base populacional nos municípios de Caracol, Piauí, e Garrafão do Norte, Pará, Brasil}

\author{
Child health in poor areas: findings from a \\ population-based study in Caracol, Piauí, \\ and Garrafão do Norte, Pará, Brazil
}

Juraci A. Cesar 1,2

Maria Aurora D. Chrestani 3

Everton J. Fantinel 3

Tatiane S. Gonçalves 4

Nelson A. Neumann 5

\footnotetext{
${ }^{1}$ Faculdade de Medicina, Universidade Federal do Rio Grande, Rio Grande, Brasil. 2 Programa de Pós-graduação em Epidemiologia, Universidade Federal de Pelotas, Pelotas, Brasil. 3 Departamento de Medicina Social, Universidade Federal de Pelotas, Pelotas, Brasil. ${ }^{4}$ Secretaria de Assistência Social, Prefeitura Municipal de Paulo Afonso, Paulo Afonso, Brasil.

${ }_{5}$ Coordenação Nacional da Pastoral da Criança, Curitiba, Brasil.

Correspondência J. A. Cesar

Programa de Pós-graduação em Epidemiologia, Universidade Federal de Pelotas.

Rua Marechal Deodoro 1160, 3 o andar, Pelotas, RS 96020-220, Brasil. jacesar@terra.com.br
}

\begin{abstract}
The aim of this study was to evaluate child health indicators in the municipalities (counties) of Caracol, Piauí State, and Garrafão do Norte, Pará State, Brazil. Through household visits using systematic sampling, previously trained interviewers applied a standard questionnaire to mothers of under-five children, investigating socioeconomic status, housing and environmental sanitation, demographic characteristics, disease patterns, and prenatal and childbirth care. The analysis used the t-test and chi-square test to compare indicators between the two municipalities. Of the 1,728 children studied, 60\% were from families with incomes less than one monthly minimum wage (approximately U\$200), 41\% had no type of sewage treatment or disposal, 10\% of mothers reported zero prenatal visits, $30 \%$ of the children were born in the same municipality, and $30 \%$ had been taken to a pediatric consultation in the previous 3 months; 20\% had a heightfor-age deficit $\geq 2$ standard deviations. All target indicators were deficient in both the municipalities (especially in Garrafão do Norte). Expanding health care supply and improving housing and sanitation conditions are priorities in both $\mathrm{mu}$ nicipalities.
\end{abstract}

Health Status Indicators; Child Welfare; Maternal-Child Health Services
Introdução

Indicadores de saúde infantil são extremamente afetados pelas condições do meio em que a criança vive e altamente dependentes da oferta suficiente e apropriada de cuidados 1,2 . Se essas condições não forem atendidas, esses indicadores não serão bons. Este cenário se faz presente, invariavelmente, nos pequenos municípios que se localizam distante dos grandes centros urbanos, que são pobres, que apresentam estrutura em saúde insuficiente e que carecem enormemente de pessoal habilitado a pensar e oferecer saúde do ponto de vista coletivo 3 . Via de regra, o que se vê é a oferta de um serviço individualizado, curativo, paliativo, repetitivo e voltado ao atendimento de uma demanda quase infinita. Isso ocorre também em virtude de os gerenciadores locais preferirem os programas verticais do governo, que já vêm acompanhados de recursos.

Por essas razões, são extremamente raras situações em que o próprio município avalia seus indicadores de saúde ${ }^{4}$, o que permitiria estabelecer linha de base para futuras avaliações, examinar a cobertura de programas, determinar prioridades de intervenção e definir metas a serem alcançadas em um determinado período ${ }^{5}$. A sua não-ocorrência acaba reduzindo o impacto das intervenções oferecidas e levando muitos programas ao descrédito, por parte tanto dos gerenciadores, quanto dos seus usuários. 
Em 2002, avaliação realizada em 18 áreas de nove dos 32 municípios mais pobres da região Norte e Nordeste do Brasil mostrou indicadores de saúde para menores de cinco anos muito aquém do desejado. Em 2005, retornou-se às mesmas áreas e os mesmos indicadores foram novamente avaliados. Além do conhecimento materno em alguns aspectos de saúde infantil, o avanço observado foi semelhante ao encontrado nas áreas-controle, revelando que, de fato, não houve intervenção ou, se houve, esta não foi efetiva ${ }^{3}$. Nesse retorno, decidiu-se por sobreamostrar dois desses municípios, sendo um da região Norte, Garrafão do Norte, no Estado do Pará, e Caracol, no Estado do Piauí, a fim de estabelecer, de forma representativa, linha de base que possibilitasse avaliar, em nível de município, futuras intervenções em saúde.

O objetivo deste artigo é apresentar indicadores de saúde infantil em dois pequenos municípios brasileiros e definir prioridades de intervenção em cada um deles.

\section{Metodologia}

Este estudo foi conduzido nos Municípios de Garrafão do Norte, Pará, e Caracol, Piauí, entre julho e setembro de 2005. Garrafão situa-se no Nordeste do Estado do Pará, distante 244 km de Belém. Nessa ocasião, este município contava com pouco mais de 23 mil habitantes e Índice de Desenvolvimento Humano (IDH) de 0,58. O Município de Caracol localiza-se no Sul do Estado do Piauí, a $470 \mathrm{~km}$ de Teresina (capital); na ocasião, contava com cerca de nove mil habitantes e IDH de 0,59 (Instituto de Pesquisa Econômica e Aplicada. http://www.ipeadata.gov.br/ipeaweb. dll/MenuCtrl?SessionID=582418562\&Mod=SOC IAL\&Lang, acessado em 27/Ago/2007).

A população-alvo deste estudo foi constituída por todas as crianças menores de cinco anos residentes em ambos os municípios no período citado. Em virtude de o principal interesse desta investigação ter sido medir prevalências, utilizou-se um delineamento transversal, o mais adequado à obtenção desse tipo de medida 6 .

O tamanho da amostra foi baseado em taxas de prevalências variando de $15 \%$ a $50 \%$ e precisão de 2,5 pontos percentuais. A estes valores, foram acrescidos $10 \%$ para suprir eventuais perdas e recusas, o que resultou em um tamanho amostral de 1.619 crianças 7 .

Decidiu-se $a$ priori que, em Caracol, metade dos domicílios seria visitada, enquanto em Garrafão do Norte, cuja população era 2,6 vezes maior, apenas um terço seria visitado. Em um primeiro momento, cada uma das comunida- des desses municípios foi mapeada pela supervisora do trabalho de campo (T. S. G.), e todas as quadras (zona urbana) ou áreas (zona rural) foram numeradas sempre no sentido horário. A partir de um ponto inicialmente indicado em cada uma dessas quadras ou áreas, sorteava-se, de forma aleatória, o primeiro domicilio, sendo os demais escolhidos de forma sistemática, até completar todos os domicílios das áreas mapeadas. Caso houvesse alguma criança com idade entre 0 e 59 meses, tanto em Caracol, quanto em Garrafão do Norte, aplicavam-se, então, os questionários.

Foram coletadas informações quanto às características demográficas (idade materna e estado civil, cor da pele; idade e sexo da criança), sócio-econômicas (escolaridade materna e renda familiar), condições de moradia (tipo de construção, tipo de piso, número de compartimentos usados para dormir, acesso a rede de esgotos e água tratada e disponibilidade de sanitário), assistência à gestação e ao parto (número de consultas de pré-natal realizadas, mês de inicio do pré-natal, doses de vacina antitetânica recebidas, tipo de parto e de profissional que o realizou e peso ao nascer), acesso aos serviços saúde e utilização destes (ocorrência de hospitalizações nos 12 meses antecedentes à entrevista, realização de consultas médicas nos últimos três meses, cobertura vacinal básica e monitoração do crescimento), padrão de morbidade nos últimos 15 dias, de amamentação e dieta e avaliação do estado nutricional.

Embora a maioria das variáveis seja auto-explicativa, algumas delas necessitam de esclarecimentos adicionais, a saber: renda familiar - valor recebido por todos aqueles residentes no domicilio no mês imediatamente anterior ao da entrevista; cor da pele - classificada pelo entrevistador em branca, parda/mulata e preta; baixo peso ao nascer - crianças com peso de nascimento inferior a 2.500g; cobertura vacinal básica completa - crianças que receberam pelo menos três doses da vacina oral contra poliomielite e tríplice bacteriana (DPT), uma dose da vacina anti-sarampo e uma dose da BCG; vacinação contra tétano neonatal - considerou-se como vacinada a gestante que recebeu três doses de vacina antitetânica nos últimos cinco anos ou que recebeu três doses da vacina há mais de cinco anos e uma dose de reforço na última gravidez; hospitalização - permanência de pelo menos 24 horas em ambiente hospitalar nos últimos 12 meses; realização de consultas médicas - se nos últimos três meses a criança consultou com médico ou enfermeira; déficit nutricional para os indicadores peso/idade, peso/altura e altura/idade, conforme o novo padrão da Organização Mundial da Saúde (WHO 
Anthro for personal computers, http:/ / www.who. int/childgrowth/software/en/).

Para este estudo, foram selecionados e treinados nove entrevistadores, que já possuíam experiência prévia em pesquisa. Durante os quatro dias de treinamento, foi realizada a leitura do questionário e manual, treinamento de entrevistas e de técnicas antropométricas. Dentre os nove entrevistadores treinados, seis foram selecionados para atuar no estudo. Os demais permaneceram como suplentes para a eventualidade de alguma substituição ou desistência.

Os seis entrevistadores selecionados foram divididos em duplas, e cada uma foi designada para cobrir uma determinada quadra na zona urbana ou área na zona rural. Ao final de cada dia de trabalho, cada entrevistador codificava os questionários aplicados naquele dia e os repassava à coordenação para conferência inicial e envio à Universidade Federal do Rio Grande (FURG) para revisão final, dupla digitação, comparação e correção final. Todo esse processo foi realizado utilizando-se o programa Epi Info versão 6.04 (Centers for Disease Control and Prevention, Atlanta, Estados Unidos). Concluída essa etapa, os dados foram transportados para o pacote estatístico Stata, versão 9.2 (Stata Corp., College Station, Estados Unidos), onde foi realizada a análise de consistência, categorização de variáveis, obtenção de medidas de tendência central e de dispersão.

O controle de qualidade foi realizado pelos entrevistadores, pelos revisores e por meio de repetição parcial dos questionários para $10 \%$ das entrevistas, estas escolhidas de forma aleatória.

Os princípios éticos foram assegurados aos entrevistados, através da explicação da importância da sua participação na pesquisa, do direito de não participar dela, se assim desejassem, da garantia de sigilo absoluto quanto às informações prestadas e coletadas e da obtenção do consentimento por escrito para participar do estudo. Finalmente, este projeto foi aprovado pelo Comitê de Ética em Pesquisa da FURG.

\section{Resultados}

Neste estudo foram visitados 3.405 domicílios, sendo 1.446 em Caracol e 1.959 em Garrafão do Norte. Nas residências, foram encontradas 1.728 crianças, sendo 606 em Caracol e 1.122 em Garrafão do Norte. O número médio de crianças em todos os domicílios com menores de cinco anos foi 0,51, sendo 0,42 em Caracol e 0,55 em Garrafão do Norte. Deste total, foi possível obter informações sobre 1.671 delas, o que representa perdas de $3,4 \%$, sendo de $2,3 \%$ (16) em Caracol e 3,3\% (41) em Garrafão do Norte.

A Tabela 1 mostra que cerca de um terço das mães era adolescente e um quinto tinha 40 anos ou mais de idade. Dentre todas as mães estudadas, cerca de $30 \%$ não sabia ler nem escrever, sendo esta proporção 53\% maior em Garrafão do Norte. Uma em cada sete mães não completou um único ano de escolaridade, enquanto uma em cada quatro concluiu nove anos ou mais. Cerca de $60 \%$ das famílias estudadas possuíam renda mensal inferior a um salário mínimo, sendo este percentual significativamente maior em Caracol do que em Garrafão. A renda per capita média mensal foi de $\mathrm{R} \$ 60$, em Caracol, a R \$ 69, em Garrafão do Norte. Pelo menos $40 \%$ dos domicílios incluídos na amostra não possuíam qualquer tipo de sanitário, sendo este percentual substancialmente maior em Caracol (64\%) do que em Garrafão do Norte (29\%). Somente em 20\% das moradias havia rádio, televisão e geladeira. Por fim, a distância média dos domicílios ao serviço de saúde mais próximo foi de $6 \mathrm{~km}$, com importante diferença entre os municípios. Em Caracol, por exemplo, $18 \%$ da população viviam a menos de $1 \mathrm{~km}$ do serviço de saúde mais próximo, contra $52 \%$ em Garrafão.

A Tabela 2 mostra que a média de idade das crianças estudadas foi 30,5 meses, estando estas uniformemente distribuídas entre os sexos. Oito em cada dez delas tinham cor da pele morena/ parda.

A assistência ao pré-natal e ao parto é sumarizada na Tabela 3. Pelo menos nove em cada dez gestantes iniciaram o pré-natal, cerca de metade ainda no primeiro trimestre. Para esta variável, houve diferença significativa entre os municípios: enquanto $83 \%$ foram à consulta médica no primeiro trimestre em Caracol, o mesmo ocorreu para somente 38\% em Garrafão. Apenas 40\% das gestantes realizaram seis ou mais consultas durante todo o pré-natal, sendo este percentual 2,5 vezes maior em Caracol (66\%) do que em Garrafão (27\%). Somente $40 \%$ disseram-se imunizadas contra tétano neonatal, sendo esta cobertura de $50 \%$ em Caracol e 35\% em Garrafão. Cerca de um quarto das crianças nasceu no próprio domicílio, sendo este percentual 3,7 vezes maior em Garrafão (35\%) do que em Caracol (9,5\%). Finalmente, $80 \%$ de todas as crianças em ambos os municípios nasceram de parto normal.

A Tabela 4 mostra que a proporção de crianças hospitalizada nos 12 meses anteriores à entrevista foi 2,3 vezes maior em Caracol (18\%) do que em Garrafão do Norte (8\%). Em Caracol, a grande maioria das crianças passou por consulta com médico (90\%), enquanto em Garrafão do Norte elas consultaram predominantemente 
Tabela 1

Características demográficas das mães, condições de moradia e distância dos serviços de saúde, para crianças menores de cinco anos residentes nos municípios de Caracol, Piauí, e Garrafão do Norte, Pará, Brasil, 2005.

\begin{tabular}{|c|c|c|c|c|}
\hline \multirow[t]{2}{*}{ Variável } & \multicolumn{2}{|c|}{ Município } & \multirow[t]{2}{*}{ Total (\%) } & \multirow[t]{2}{*}{ Valor de $p$ * } \\
\hline & Caracol (\%) & Garrafão do Norte (\%) & & \\
\hline Idade em anos completos & & & & $<0,001$ \\
\hline Menos de 20 & 31,2 & 33,6 & 32,0 & \\
\hline $20-29$ & 28,9 & 38,3 & 32,2 & \\
\hline $30-39$ & 16,9 & 16,3 & 16,7 & \\
\hline 40 ou mais & 23,0 & 11,9 & 19,1 & \\
\hline Idade em anos: média (desvio-padrão) & $23,4(6,9)$ & $25,2(8,7)$ & $24,6(8,1)$ & $<0,001 * \star$ \\
\hline Não sabem ler nem escrever & 24,4 & 37,5 & 29,0 & $<0,001$ \\
\hline \multicolumn{5}{|l|}{ Anos completos de escolaridade da mãe } \\
\hline Nenhum & 12,9 & 17,6 & 14,6 & $<0,001$ \\
\hline $1-3$ & 23,9 & 22,7 & 23,5 & \\
\hline $4-8$ & 37,7 & 38,8 & 38,2 & \\
\hline 9 ou mais & 25,4 & 20,8 & 23,8 & \\
\hline Escolaridade em anos: média (desvio-padrão) & $4,7(3,6)$ & $5,1(3,5)$ & $4,9(3,5)$ & 0,061 ** \\
\hline Renda familiar mensal em salários mínimos & $(n=587)$ & $(n=1050)$ & $(n=1637)$ & \\
\hline Menos de 1 & 69,0 & 52,5 & 58,4 & $<0,001$ \\
\hline 1,9 & 16,3 & 25,5 & 22,2 & \\
\hline 2 ou mais & 14,6 & 22,0 & 19,4 & \\
\hline Renda familiar em salários mínimos: média (desvio-padrão) & $1,0(1,4)$ & $1,4(1,7)$ & $1,3(1,6)$ & $<0,001 * *$ \\
\hline Número de moradores por domicílio: média (desvio-padrão) & $5,0(2,0)$ & $6,1(2,6)$ & $5,7(2,5)$ & $<0,001$ ** \\
\hline \multicolumn{5}{|l|}{ Tipo de construção da moradia } \\
\hline Tijolo/Bloco & $0,0 \%$ & 27,8 & 18,0 & $<0,001$ \\
\hline Taipa & 1,0 & 26,1 & 17,2 & \\
\hline Madeira & 98,0 & 30,1 & 56,4 & \\
\hline Outro & 1,0 & 15,2 & 10,2 & \\
\hline Tipo de sanitário & & & & $<0,001$ \\
\hline Com descarga & 11,5 & 12,3 & 12,0 & \\
\hline Sem descarga & 19,7 & 12,5 & 15,0 & \\
\hline Casinha/Fossa negra & 5,2 & 45,9 & 31,5 & \\
\hline Não tem & 63,6 & 29,3 & 41,4 & \\
\hline \multicolumn{5}{|l|}{ Origem da água usada para beber } \\
\hline Rede pública, mas sem tratamento & 2,0 & 33,1 & 22,1 & $<0,001$ \\
\hline Cisterna/Poço & 46,1 & 59,0 & 54,5 & \\
\hline Rio/Lago/Lagoa/Açude & 22,5 & 0,6 & 8,3 & \\
\hline Outros & 29,32 & 7,31 & 15,1 & \\
\hline Domicílios com rádio, televisão e geladeira & 20,5 & 20,0 & 20,2 & 0,833 \\
\hline Distância do serviço de saúde mais próximo $(\mathrm{km})$ & & & & $<0,001$ \\
\hline Menos de 1 & 17,6 & 52,1 & 39,9 & \\
\hline $1-4,9$ & 30,5 & 19,7 & 23,5 & \\
\hline 5 ou mais & 51,9 & 28,2 & 36,6 & \\
\hline Distância em km: média (desvio-padrão) & $6,0(5,9)$ & $6,0(12,9)$ & $6,1(11,8)$ & $1,00 * \star$ \\
\hline Total $(n)$ & 590 & 1081 & 1671 & \\
\hline
\end{tabular}

* Teste de qui-quadrado de heterogeneidade;

** Teste t.

com enfermeira (76\%). Em ambos os municípios, segundo relato das mães, quase não houve consulta preventiva (puericultura). Por fim, com base no novo padrão OMS (Organização Mundial da Saúde), o déficit de altura para a idade nas formas moderada e grave $(\leq-2$ desvios-padrão $-D P)$ atingia em torno de um quarto das crianças de Garrafão do Norte. Cerca de $2 \%$ de todas as crian- 
Características demográficas de crianças menores de cinco anos residentes nos municípios de Caracol, Piauí, e Garrafão do Norte, Pará, Brasil, 2005

\begin{tabular}{|c|c|c|c|c|}
\hline \multirow[t]{2}{*}{ Variável } & \multicolumn{2}{|c|}{ Município } & \multirow[t]{2}{*}{ Total (\%) } & \multirow[t]{2}{*}{ Valor de $p$ * } \\
\hline & Caracol (\%) & Garrafão do Norte (\%) & & \\
\hline Idade da criança (em meses) & & & & 0,695 \\
\hline $0-5$ & 11,2 & 9,7 & 10,2 & \\
\hline $6-11$ & 7,3 & 8,9 & 8,3 & \\
\hline $12-23$ & 20,0 & 18,9 & 19,1 & \\
\hline $24-35$ & 19,8 & 21,4 & 20,9 & \\
\hline $36-47$ & 22,7 & 22,9 & 22,9 & \\
\hline $28-59$ & 18,9 & 18,5 & 18,7 & \\
\hline Idade em meses: média (desvio-padrão) & $30,4(17,0)$ & $30,5(17,0)$ & $30,5(17,0)$ & 0,895 ** \\
\hline \multicolumn{5}{|l|}{ Sexo } \\
\hline Masculino & 50,3 & 48,6 & 49,2 & 0,488 \\
\hline Feminino & 49,7 & 51,4 & 50,8 & \\
\hline Cor da pele & & & & $<0,001$ \\
\hline Branca & 10,0 & 14,1 & 12,7 & \\
\hline Morena/Parda & 80,0 & 84,1 & 82,6 & \\
\hline Preta & 10,0 & 1,8 & 4,7 & \\
\hline Total (n) & 590 & 1.081 & 1.671 & \\
\hline
\end{tabular}

* Teste de qui-quadrado de heterogeneidade;

** Teste t.

ças estudadas apresentavam-se com sobrepeso ou obesas $(\geq+2 \mathrm{DP})$

Finalmente, a Tabela 5 apresenta o padrão de utilização de serviços preventivos em saúde e recebimento de auxílio do Programa Bolsa Família. Cerca de três quartos das crianças com idade entre 12 e 23 meses haviam recebido o esquema vacinal básico completo. Ao se analisarem crianças com idade entre 12 e 59 meses, a proporção aumentou para $85 \%$. Uma em cada cinco crianças estava recebendo o benefício do Programa Bolsa Família, sendo a cobertura ligeiramente maior em Caracol (26\%) do que em Garrafão do Norte $(20 \%)$. Vale destacar que essa cobertura refere-se a meados de 2005 .

\section{Discussão}

Os resultados obtidos mostram tratar-se de uma população com enormes necessidades em termos sócio-econômicos, habitacionais e de assistência em saúde. Revelou-se também elevada proporção de mães adolescentes, nível de escolaridade baixo, renda familiar insuficiente, saneamento básico precário, assistência inadequada ao parto e elevada prevalência de morbidade, sobretudo de déficit nutricional para o indicador altura/idade, e inexistência de consultas de rotina (puericultura). Praticamente todos os indicadores de morbidade e de utilização de serviços de saúde foram, de forma sistemática, piores em Garrafão do Norte, em relação a Caracol.

O delineamento transversal, embora não permita avaliar causalidade, possibilita a contagem dos eventos observados. Essa quantificação permite: (i) conhecer a magnitude de cada um dos indicadores (ou variáveis) estudados; (ii) avaliar a cobertura de programas que atendem uma determinada população; (iii) definir prioridades de intervenção; (iv) estabelecer linha de base sobre os indicadores estudados a fim de avaliar impacto de futuras intervenções em saúde 5,6. Daí a razão da sua utilização neste estudo.

A realização de pelo menos seis consultas de pré-natal e de exames laboratoriais e clínicos básicos, associados a adequado atendimento em sala de parto, possibilitaria redução de até um quarto das mortes infantis e da quase totalidade dos óbitos maternos 1 .

Os indicadores relativos à assistência à gestação e ao parto observado nos dois municípios estão muito aquém do minimamente desejado e daqueles valores verificados em áreas urbanas 8,9. Há clara necessidade de captar mais gestantes, especialmente no início do pré-natal. 
Padrão de utilização de serviços de saúde e de morbidade, entre crianças menores de cinco anos residentes nos municípios de Caracol, Piauí, e Garrafão do Norte, Pará, Brasil, 2005

\begin{tabular}{|c|c|c|c|c|}
\hline \multirow[t]{3}{*}{ Variável } & \multicolumn{2}{|c|}{ Município } & \multirow[t]{3}{*}{ Total } & \multirow{3}{*}{$\begin{array}{l}\text { Valor } \\
\text { de } p \text { * }\end{array}$} \\
\hline & Caracol (\%) & & & \\
\hline & & do Norte (\%) & & \\
\hline Hospitalizados nos últimos 12 meses & 18,5 & 8,0 & 11,7 & $<0,001$ \\
\hline Causa da hospitalização ** & $(n=590)$ & $(n=1.081)$ & $(n=1.671)$ & \\
\hline Diarréia & 11,0 & 3,1 & 5,9 & $<0,001$ \\
\hline Pneumonia & 3,9 & 1,1 & 2,1 & $<0,001$ \\
\hline Outra & 10,2 & 5,7 & 7,3 & $<0,001$ \\
\hline Foi levada à consulta médica nos últimos três meses & 29,8 & 29,0 & 29,3 & 0,737 \\
\hline Número de vezes que consultou com & $(n=176)$ & $(n=314)$ & $(n=490)$ & \\
\hline Médico & 89,8 & 27,7 & 50,0 & $<0,001$ \\
\hline Enfermeira & 12,6 & 76,1 & 53,3 & $<0,001$ \\
\hline \multicolumn{5}{|l|}{ Motivo da consulta médica } \\
\hline Diarréia & 5,2 & 5,2 & 5,2 & 0,948 \\
\hline Pneumonia & 1,5 & 1,2 & 1,3 & 0,580 \\
\hline Desnutrição & 0,0 & 0,4 & 0,2 & 0,139 \\
\hline Doenças de pele & 0,7 & 0,9 & 0,8 & 0,596 \\
\hline Acidentes & 0,4 & 0,3 & 0,4 & 0,919 \\
\hline Infecção respiratória alta & 11,0 & 8,7 & 9,5 & 0,122 \\
\hline Puericultura (rotina) & 0,5 & 0,7 & 0,7 & 0,576 \\
\hline Déficit de peso para a idade/OMS & & & & 0,212 \\
\hline Severo $(\leq-3 \mathrm{DP})$ & 0,3 & 0,7 & 0,5 & \\
\hline Moderado (-2 DP a -2,9 DP) & 4,8 & 5,4 & 5,2 & \\
\hline Leve (-1 DP a -1,9 DP) & 17,9 & 22,1 & 20,6 & \\
\hline Normal ( $\geq 0,99 \mathrm{DP}$ a +1,99 DP) & 77,0 & 71,8 & 73,7 & \\
\hline \multicolumn{5}{|l|}{ Déficit de altura para a idade/OMS } \\
\hline Severo $(\leq-3 \mathrm{DP})$ & 4,2 & 6,5 & 5,7 & $<0,001$ \\
\hline Moderado (-2 DP a -2,9 DP) & 9,2 & 16,8 & 14,0 & \\
\hline Leve (-1 DP a -1,9 DP) & 25,5 & 31,9 & 29,6 & \\
\hline Normal ( $\geq-0,99 \mathrm{DP})$ & 61,1 & 44,8 & 50,6 & \\
\hline \multicolumn{5}{|l|}{ Déficit de peso para altura/OMS } \\
\hline Severo ( $\leq-3 \mathrm{DP})$ & 0,2 & 0,0 & 0,1 & \\
\hline Moderado (-2 DP a -2,9 DP) & 0,6 & 1,9 & 1,0 & $<0,050$ \\
\hline Leve (-1 DP a -1,9 DP) & 8,9 & 11,8 & 9,9 & \\
\hline Normal ( $\geq-0,99 \mathrm{DP})$ & 87,9 & 84,2 & 86,6 & \\
\hline Sobrepeso/obeso ( $\geq+2 \mathrm{DP})$ & 2,4 & 2,1 & 2,3 & \\
\hline Total (n) & 590 & 1.081 & 1.671 & \\
\hline
\end{tabular}

DP: desvio-padrão; OMS: Organização Mundial da Saúde.

* Teste de qui-quadrado de heterogeneidade;

** Excede ao total geral em virtude de uma mesma criança poder ter sido hospitalizada por diferentes causas.

Realizar busca ativa de gestantes na comunidade seria uma solução, mas somente em Caracol, pela menor dificuldade de acesso, menor extensão territorial e pela presença de agentes comunitários de saúde (ACS), diferentemente do que ocorre em Garrafão do Norte, onde esses fatores são desfavoráveis. Nesta cidade, a única solução seria expandir a rede local de saúde por meio da construção de unidades básicas de saúde nas comunidades mais populosas, contratação de profissionais e facilitação de transporte de comunidades menores até estes locais. Em Caracol, embora percentual elevado de mães inicie o pré-natal no primeiro trimestre, há necessidade de criar estratégias visando a aumentar a proporção daquelas que realizam pelo menos 
Padrão de utilização de serviços preventivos em saúde e recebimento de auxílio do Programa Bolsa Família, entre crianças menores de cinco anos residentes nos municípios de Caracol, Piauí, e Garrafão do Norte, Pará, Brasil, 2005.

\begin{tabular}{|c|c|c|c|c|}
\hline \multirow[t]{2}{*}{ Variável } & \multicolumn{2}{|c|}{ Município } & \multirow[t]{2}{*}{ Total } & \multirow[t]{2}{*}{ Valor de $p$ * } \\
\hline & Caracol (\%) & Garrafão do Norte (\%) & & \\
\hline Verificação do peso da criança nas últimas quatro semanas ** & 61,9 & 35,6 & 44,9 & $<0,001$ \\
\hline Posse do cartão da criança & & & & $<0,005$ \\
\hline Sim, visto & 96,3 & 92,8 & 94,0 & \\
\hline Sim, não visto & 3,2 & 3,8 & 3,6 & \\
\hline Tinha, mas perdeu & 0,2 & 0,7 & 0,5 & \\
\hline Não, nunca teve & 0,3 & 2,7 & 1,9 & \\
\hline \multicolumn{5}{|l|}{ Imunização completa dos 12 aos 23 meses de idade contra } \\
\hline Poliomielite (Sabin) & 99,0 & 92,4 & 94,8 & $<0,050$ \\
\hline Difteria, tétano e coqueluche (DPT) & 97,0 & 77,3 & 84,3 & $<0,001$ \\
\hline Sarampo (anti-sarampo) & 91,9 & 76,2 & 81,8 & 0,001 \\
\hline Tuberculose (BCG) & 99,0 & 96,2 & 97,2 & 0,171 \\
\hline Cobertura vacinal básica completa (3 doses de Sabin e DPT, & 90,9 & 63,5 & 73,2 & $<0,001$ \\
\hline \multicolumn{5}{|l|}{1 dose de anti-sarampo e BCG) } \\
\hline \multicolumn{5}{|l|}{ Imunização completa dos 12 aos 59 meses de idade contra } \\
\hline Poliomielite (Sabin) & 99,6 & 96,0 & 97,3 & $<0,001$ \\
\hline Difteria, tétano e coqueluche (DPT) & 97,7 & 84,0 & 88,8 & $<0,001$ \\
\hline Sarampo (anti-sarampo) & 96,8 & 91,2 & 93,2 & $<0,001$ \\
\hline Tuberculose (BCG) & 99,8 & 97,2 & 98,1 & 0,001 \\
\hline Cobertura vacinal básica completa (3 doses de Sabin e DPT, & 94,8 & 79,3 & 84,8 & $<0,001$ \\
\hline \multicolumn{5}{|l|}{1 dose de anti-sarampo e BCG) } \\
\hline Recebem auxílio do Programa Bolsa Família & 25,6 & 19,6 & 21,7 & $<0,001$ \\
\hline Total & 590 & 1.081 & 1.671 & \\
\hline
\end{tabular}

* Teste de qui-quadrado de heterogeneidade;

** Crianças menores de dois anos.

seis consultas. Além da busca ativa pelos ACS, poderiam ser criados mecanismos de incentivo, tais como pagamento de transporte até o serviço de saúde, auxílio na preparação do enxoval do recém-nascido, entre outros.

Dois outros aspectos chamam ainda a atenção em relação ao conjunto de indicadores observados: (i) o fato de, mesmo entre municípios muito pobres, haver diferenças significativas para muitos dos indicadores estudados e (ii) de o Município de Garrafão do Norte, com melhor nível sócio-econômico, apresentar piores indicadores em relação a Caracol. No primeiro caso, as diferenças podem ser atribuídas a aspectos geográficos como distância desses municípios de centros urbanos maiores, dificuldade de acesso, baixa densidade populacional, infra-estrutura insuficiente, dificuldade de contratar profissionais, gerenciamento deficiente do setor saúde, ou emancipação em momentos diferentes. No segundo caso, seria esperado que os indicadores de assistência à saúde fossem piores em Caracol, uma vez que este município está localizado no semi-árido Nordestino, a região mais pobre do país e referida como detentora dos piores indicadores de saúde infantil. Mas não foi o que se observou.

Isso pode decorrer de comparações inadequadas feitas ao longo dos anos com a Região Norte, onde, por dificuldade de acesso, muitos dados provêm de estimativas feitas para municípios mais populosos, dando a falsa idéia de que municípios menores apresentam indicadores semelhantes aos de municípios maiores, em geral, melhores. Este estudo não foi afetado por essa limitação porque incluiu, de forma representativa, não somente um município com baixa densidade populacional, mas, também, a sua população rural.

A realização de parto domiciliar por pessoal não capacitado e a baixa cobertura de imunização contra tétano neonatal são situações também preocupantes. $\mathrm{O}$ atendimento ao parto por pessoal adequadamente treinado é umas das 
medidas de maior impacto sobre a morbimortalidade materno-infantil, e a imunização contra tétano neonatal é a única forma efetiva de evitar a doença no recém-nascido ${ }^{1}$. Análise adicional desses dados mostrou, como se esperava, que a ocorrência de parto domiciliar foi cerca de duas vezes maior entre crianças cujas famílias possuíam renda inferior a um salário mínimo mensal, quando comparadas àquelas que recebiam dois salários mínimos mensais ou mais, e de quatro vezes ao se compararem mães não alfabetizadas com mães que tinham oito anos ou mais de escolaridade. Soma-se a isso o fato de $2 \%$ de todas as mães terem dado à luz sem qualquer tipo de assistência. Portanto, melhorar as condições de atendimento ao parto deve ser prioritário nessas localidades, sobretudo em Garrafão do Norte.

O Brasil vive uma epidemia de cesarianas, com prevalência que varia de $40 \%$ entre usuárias do SUS a quase $90 \%$ entre usuárias de serviços privados e/ou conveniados de saúde (Departamento de Informática do SUS. Indicadores e dados básicos do Brasil, 2004-2007. http://www. datasus.gov.br/idb, acessado em 28/Jun/2008), quando o recomendável é um percentual ao redor de $15 \%$ 10. Isso decorre de interesses não só das gestantes, como também dos prestadores de serviços 11 , porém o fato é que, para a grande maioria das gestantes, esse tipo de intervenção é desnecessário.

Analisando-se somente a freqüência de cesarianas neste estudo, seria possível afirmar que os valores observados estão dentro do recomendado. Embora não tenham sido coletadas informações quanto a risco gestacional biológico, é possível sugerir que essa aparente adequação esconde enorme iniqüidade. Análise adicional mostrou que a ocorrência de cesarianas foi cerca de três vezes maior entre as mães mais ricas (2 salários mínimos mensais ou mais), em relação às demais, e que, em relação à escolaridade, essa diferença foi dez vezes maior quando se comparam mães que têm oito anos ou mais de escolaridade com aquelas de menor escolaridade. Considerando que as mais pobres são as que apresentam, em geral, os maiores riscos, é de se supor que muitas delas não estejam recebendo o benefício dessa intervenção, enquanto outras, de menor risco, estejam fazendo uso dele sem necessidade.

Diarréia e pneumonia, doenças comuns, de fácil manejo, foram as principais causas de utilização de serviços de saúde nos dois municípios. Embora progressos expressivos tenham sido obtidos em relação a essas enfermidades, sobretudo à diarréia, ambas respondem ainda por cerca de dois terços dos óbitos infantis nos países em desenvolvimento ${ }^{12}$. A ampla disponibilidade $\mathrm{e}$ utilização do soro reidratante oral foi o principal responsável pela queda da taxa de mortalidade infantil no Brasil nas últimas décadas 13 , todavia, ainda que os óbitos por pneumonia tenham diminuído no país como um todo, trata-se uma doença de mais difícil diagnóstico, tratamento e manejo. Por isso, esforços precisam ser feitos nessas localidades, com destaque para o incentivo ao aleitamento materno, fator comprovadamente protetor contra pneumonia 14; melhoria no abastecimento de água tratada nos domicílios; ampliação da rede básica de saúde e capacitação da equipe local de saúde na estratégia de Atenção às Doenças mais Prevalentes na Infância (AIDPI), a fim de promover o manejo adequado dessas doenças.

Praticamente não houve consulta de puericultura nos três meses antecedentes à entrevista nos municípios estudados. Isso pode ser resultado da baixa valorização desse tipo de cuidado em saúde e/ou da incapacidade de a infra-estrutura disponível atender toda a demanda existente, que acaba priorizando a oferta de cuidados curativos.

Os indicadores nutricionais obtidos mostram tratar-se de uma população cuja ingestão de alimentos é insuficiente para a maioria deles, haja vista que $13 \%$ das crianças estudadas em Caracol e $23 \%$ em Garrafão apresentavam déficit para o indicador altura/idade inferior a -2 DP. Ao mesmo tempo, cerca de $2 \%$ deles já apresentavam sobrepeso ou obesidade $(\geq+2 \mathrm{DP})$. A coexistência desses dois extremos em uma mesma população traz um novo desafio, o de lidar em um mesmo espaço geográfico com problemas de determinantes opostos e diferentes conseqüências. Considerando que, nessa situação, o estabelecimento de medidas coletivas tende a agravar um dos quadros 1, a abordagem deverá ser individualizada. Para isso, há necessidade de aumentar a oferta de serviços de saúde, capacitar profissionais ao manejo adequado desses problemas, promover educação em saúde e a prevenção de doenças junto às mães e, por fim, atuar em parceria com outros setores públicos, tais como assistência social e educação.

Assim como outros indicadores, a monitoração do crescimento e a cobertura vacinal, esta para diferentes idades, ficaram muito aquém do observado em outras localidades 4,8,9. Em virtude da pouca importância dada a atividades preventivas nos dois municípios de que trata este estudo, vide a realização de puericultura, não se poderia esperar resultado diferente. No entanto, merece destaque o fato de a quase totalidade das crianças possuir cartão de saúde, sem que este seja utilizado. Isto sugere baixa valorização deste tipo de procedimento e revela a incapacidade de 
os serviços locais de saúde atenderem a demanda que têm, optando pela prestação de serviços curativos. No caso da cobertura vacinal, essa desvalorização foi menos evidente, sobretudo em Caracol. Isto se deve a intervenção especifica decorrente de estudo preliminar realizado em algumas áreas daquele município em 2002 3, que deu prioridade à vacinação básica de crianças que viessem aos serviços de saúde e de realizar busca ativa nos domicílios, sobretudo em época de campanha de multivacinação, através dos ACS e de um profissional que dispunha de uma motocicleta para deslocar-se até os domicílios e realizar vacinação.

\section{Resumo}

Este estudo teve por objetivo conhecer indicadores básicos de saúde infantil nos municípios de Caracol, Piauí, e Garrafão do Norte, Pará, Brasil. Mediante visitas domiciliares por amostragem sistemática, aplicaram-se questionários às mães de menores de cinco anos, investigando nível sócio-econômico, condições de habitação e saneamento da família, características demográficas, padrão de morbidade, de utilização de serviços de saúde e de assistência recebida durante a gestação e o parto. A análise consistiu da comparação de indicadores entre os dois municípios e foi avaliada através dos testes te qui-quadrado. Das 1.728 crianças estudadas, $60 \%$ de suas famílias possuíam renda inferior a um salário mínimo mensal; $41 \%$ não contavam com qualquer tipo de sanitário; $10 \%$ de suas mães não realizaram uma única consulta de pré-natal; $30 \%$ nasceram no domicilio; $30 \%$ foram levadas à consulta médica nos últimos três meses; $20 \%$ apresentavam déficit $\geq 2$ desvios-padrão para o indicador alturalidade. Todos os indicadores estudados foram ruins em ambos os municipios, sobretudo em Garrafão do Norte. Aumentar a oferta de cuidados em saúde e melhorar as condições de habitação e saneamento deveriam ser prioridades nessas localidades.

Indicadores Básicos de Saúde; Bem-Estar da Criança; Serviços de Saúde Materno-Infantil
Por fim, este estudo demonstra a importância de diagnósticos de saúde como o aqui realizado com o objetivo de, sobretudo, (i) conhecer indicadores básicos de saúde infantil, (ii) definir prioridades de intervenção e criar condições para avaliação da evolução temporal desses indicadores. Mostra também a necessidade de, mesmo entre os municípios mais pobres, estudar iniqüidades em saúde. O conhecimento pormenorizado dessas diferenças pode facilitar a adoção de medidas mais específicas, que alcancem maior impacto sobre essas populações e, conseqüentemente, melhorem indicadores de saúde materno-infantil nessas localidades.

\section{Colaboradores}

J. A. Cesar foi responsável pelo delineamento, análise de dados e redação final. M. A. D. Chrestani e E. J. Fantinel auxiliaram na análise de dados e redação final. T.S. gonçalves supervisionou a coleta de dados e participou da redação final. N. A. Neumann participou da definição do delineamento e auxiliou na análise de dados e redação final.

\section{Agradecimentos}

Às mães, crianças e secretarias municipais de saúde dos dois municípios, pela participação neste estudo; ao Fundo das Nações Unidas para a Infância, à Pastoral da Criança e ao Programa Criança Esperança da Rede Globo de Televisão, por terem apoiado a sua realização. 


\section{Referências}

1. Victora CG, Cesar JA. Saúde materno-infantil no Brasil: padrões de morbidade e possíveis intervenções. In: Rouquayrol MZ, Almeida-Filho NM, organizadores. Epidemiologia e saúde. 6a Ed. Rio de Janeiro: Editora MEDSI; 2003. p. 415-67.

2. Kerr-Pontes LR, Rouquayrol MZ. Medida da saúde coletiva. In: Rouquayrol MZ, Almeida-Filho NM. Epidemiologia e saúde. $6^{\text {a }}$ Ed. Rio de Janeiro: Editora MEDSI; 2003. p. 37-82.

3. Cesar JA, Gonçalves TS, Neumann N, OliveiraFilho JA, Diziekaniak AC. Saúde infantil em áreas pobres das Regiões Norte e Nordeste do Brasil: comparando indicadores básicos em áreas atendidas pela Pastoral da Criança e áreas-controle. Cad Saúde Pública 2005; 21:1845-55.

4. Cesar JA, Cavaleti MA, Holthausen RS, Lima GS. Mudança em indicadores de saúde infantil em um município com agentes comunitários: o caso de Itapirapuã Paulista, Vale do Ribeira, São Paulo, Brasil. Cad Saúde Pública 2002; 18:1647-54.

5. Vaughan JP, Morrow R. Epidemiologia para municípios: manual para gerenciamento dos distritos sanitários. São Paulo: Editora Hucitec; 1992.

6. Silva IS. Cancer epidemiology: principles and methods. Lyon: World Health Organization/International Agency for Research on Cancer; 1999.

7. Dean AG, Dean JA, Coulombier D, Brendel KA, Smith DC, Burton AH, et al. Epi-Info, version 6: a word processing, database, and statistics program for epidemiology on microcomputers. Atlanta: Centers of Disease Control and Prevention; 2002.
8. Cesar JA, Mendoza-Sassi R, Horta BL, Ribeiro PRP, D'Avila AC, Santos FM, et al. Basic indicators of child health in an urban area in southern Brazil: estimating prevalence rates and evaluating differentials. J Pediatr (Rio J) 2006; 82:437-44.

9. Cesar JA, Matijasevich AM, Santos IS, Barros AJD, Dias-da-Costa JD, Barros FC, et al. The use of maternal and child health services in three population-based cohorts in Southern Brazil, 1982-2004. Cad Saúde Pública 2008; 24 Suppl 3:S427-36.

10. World Health Organization. Appropriate technology for birth. Lancet 1985; 2:436-7.

11. Béhague DP, Victora CG, Barros FC. Consumer demand for caesarean sections in Brazil: informed decision making, patient choice, or social inequality? A population based birth cohort study linking ethnographic and epidemiological methods. BMJ 2002; 324:942-5.

12. Black RE, Morris SS, Bryce J. Where and why are 10 million children dying every year? Lancet 2003; 361:2226-34.

13 Victora CG, Olinto MT, Barros FC, Nobre LC. Falling diarrhoea mortality in Northeastern Brazil: did ORT play a role? Health Policy Plan 1996; 11: 132-41.

14. Cesar JA, Victora CG, Barros FC, Santos IS, Flores JA. Impact of breast feeding on admission for pneumonia during postneonatal period in Brazil: nested case-control study. BMJ 1999; 318:1316-20.

Recebido em 04/Jul/2008

Versão final reapresentada em 11/Nov/2008

Aprovado em 08/Dez/2008 